\title{
Cálculo de la tenacidad de fractura a través de ensayos dinámicos ${ }^{(\bullet)}$
}

\author{
F.J. Perosanz ${ }^{(*)}$, M. Serrano ${ }^{(*)}$, C. Martínez ${ }^{(*)}$ y J. Lapeña ${ }^{(*)}$
}

Resumen Uno de los componentes críticos de una central nuclear es la vasija del reactor, debido a su función de contención del núcleo. Dicha vasija está sometida a irradiación neutrónica, lo que provoca cambios microestructurales en el material y pérdida de propiedades mecánicas. Debido a estos efectos, es necesario monitorizar su integridad estructural a lo largo de su vida de operación. Para ello se establecen los llamados programas de vigilancia. El objetivo final de estos ensayos es el de determinar la tenacidad de fractura del material. Actualmente, esto se consigue indirectamente mediante técnicas de predicción establecidas en diferentes normativas. El objetivo de este trabajo es el de determinar la tenacidad de fractura del material de la vasija directamente a través del ensayo Charpy V instrumentado. Para ello se ha desarrollado en el CIEMAT una metodología de ensayos y análisis de resultados.

Palabras clave: Irradiación neutrónica. Tenacidad de fractura. Charpy instrumentado. Vasija del reactor. Reactores $L W R$.

\section{Fracture toughness calculation using dynamic testing}

\begin{abstract}
The most critical component of a Nuclear Power Station is the Reactor Pressure Vessel (RPV), due to safety and integrity requirements. The RPV is subjected to neutron radiation and this phenomenon lead to microstructural changes in the material and modifications in the mechanical properties. Due to this effects, it is necessary to assess the structural integrity of the RPV along the operational life through surveillance programs. The main objetive of this surveillance programs is to determine the fracture toughness of the material. At present this objetive is reached combining direct measures and prediction techniques. In this work, direct measures of fracture toughness using instrumented Charpy V impact testing are present using a CIEMAT development on analysis of results.
\end{abstract}

Keywords: Neutron embrittlement. Fracture toughness. Instrumented impact testing. Reactor pressure vessel. $L W R$ reactors.

\section{INTRODUCCIÓN}

Uno de los componentes críticos de una central nuclear es la vasija del reactor, debido a su función de contención del núcleo. Presión, temperatura y estado del material son las variables esenciales a tener en cuenta para controlar la integridad estructural de la vasija. Las dos primeras pueden ser monitorizadas en continuo por lo que su evaluación es directa, mientras que el estado del material viene condicionado por la fluencia neutrónica recibida y esta variable no es medible de forma continua.

(•) Trabajo recibido el día 20 de noviembre de 1997 y aceptado en su forma final el 24.3.98.

(*) Instituto de Tecnología Nuclear, CIEMAT. Centro de Investigaciones Energéticas Medioambientales y Tecnológicas. Avda. Complutense, 22. 28040-Madrid (España).
Siguiendo la teoría de la mecánica de fractura elástico lineal, los valores de presión y temperatura de operación de la vasija del reactor deben ser tales que no originen un valor de $K_{\mathrm{I}}$ (factor de intensidad de tensiones) que alcancen el valor de $K_{\text {IC }}$ (tenacidad de fractura del material) para un tamaño de defecto postulado, lo que originaría la rotura frágil del material. El cálculo de $K_{\mathrm{I}}$, función de $P$ y $T$, está establecido en el Código ASME para un tamaño de defecto postulado, con lo cual lo que se debe de calcular periódicamente es el valor de tenacidad de fractura, $K_{\mathrm{IC}}$, del material.

Un diagrama típico, $P-T$, con zonas de operación se muestra en la figura 1. Debido al daño producido por la irradiación neutrónica, la curva crítica inicial evoluciona con el tiempo, es decir, el valor de $K_{\text {IC }}$ desplazándose de izquierda a derecha y reduciendo la zona útil de operación normal (área rayada de la figura). 


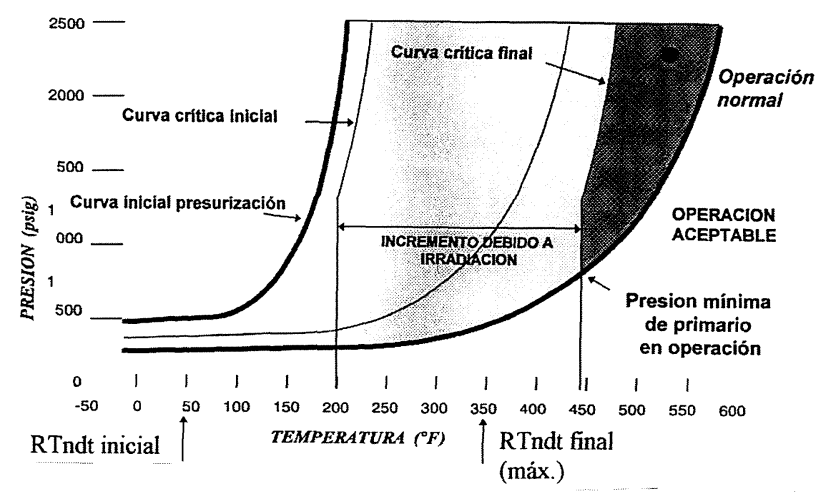

FIG. 1.- Curva de operación.

FIG. 1.-Operating curve.

De todo lo expuesto anteriormente, se deduce que la integridad estructural de la vasija del reactor se basa en la determinación del desplazamiento del valor de la tenacidad de fractura, $K_{\mathrm{IC}}$, debida a la irradiación neutrónica.

El procedimiento actual de la determinación de $K_{\text {IC }}$ se realiza a través de los ensayos de las cápsulas de vigilancia situadas dentro de la vasija, las cuales contienen en su interior probetas (Charpy V y en algunos casos CT 1/2") pertenecientes a zonas críticas expuestas a irradiación (belt line y soldaduras) del mismo material utilizado en la fabricación de la vasija y con el mismo tratamiento termomecánico de conformado.

El ensayo Charpy V ha sido tradicionalmente incluido en los procedimientos de vigilancia para evaluar la degradación mecánica del material. Según la normativa de análisis de estas cápsulas de vigilancia se acepta que el desplazamiento que sufre la curva energía-temperatura a un nivel prefijado de $30 \mathrm{ft} / \mathrm{lb}$ ó $41 \mathrm{~J}$ es el mismo que el desplazamiento de la curva tenacidad-temperatura, tal como se señala en la figura 2.

La inclusión de un transductor de fuerza en la cuchilla del péndulo permite obtener datos de fuerza instantánea-tiempo, mediante los cuales se pueden obtener parámetros de mayor interés que la simple energía de rotura. Estos ensayos en Charpy instrumentado son la base del presente trabajo. A través de los registros típicos obtenidos carga-tiempo o carga-desplazamiento se han obtenido valores característicos del material comparables a la tenacidad de fractura estática. Esta herramienta presenta, pues, un elevado interés de cara a una evaluación más detallada de las cápsulas de vigilancia, que como se ha comentado con anterioridad es la única herramienta disponible para monitorizar el estado mecánico de la vasija en función de la irradiación neutrónica.

El objetivo principal de este trabajo es disponer de un método validado de estimación de la tenacidad de fractura directamente sobre el material irra-
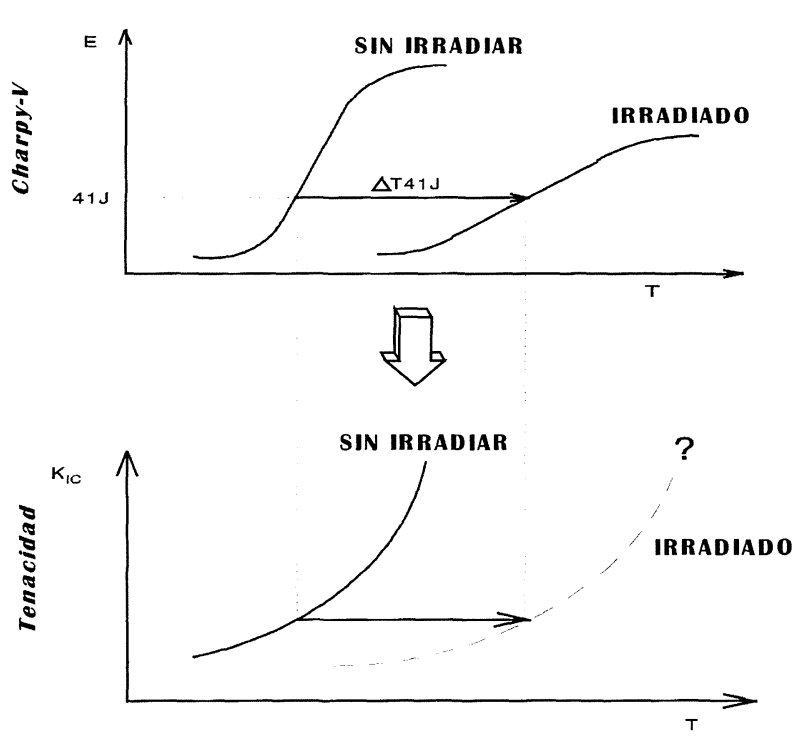

FIG. 2.- Desplazamiento de la curva de tenacidad de fractura en función del desplazamiento de la curva Charpy a $41 \mathrm{~J}$ de energía.

FIG. 2.- Fracture toughness displacement as a function of Charpy curve displacement at $30 \mathrm{ft} / \mathrm{lb}$ energy level.

diado, sin las incertidumbres añadidas de las dos suposiciones asumidas:

- El desplazamiento de la curva $K_{\mathrm{IC}}-T$ es idéntico al sufrido por la curva $E-T$ para un nivel de energía dado.

- El hábito de la curva $K_{\mathrm{IC}}-T$ se conserva, independientemente del nivel de fluencia neutrónica total recibido por el material.

\section{PARTE EXPERIMENTAL}

\subsection{Material}

En este trabajo se han empleado dos materiales; un acero típico de vasijas de presión de reactores nucleares A-533B y un acero de uso común F-156. La composición química y las principales cualidades mecánicas de estos aceros vienen reseñadas en la tabla I. La finalidad de este estudio es abarcar una amplia variedad de materiales para comprobar que son válidos los criterios empleados en la determinación y cálculo de tenacidades de fractura a través de ensayos de impacto

Para el acero A-533B, al que se denominará A, se aprecia en la figura 3 , a escasos aumentos $(\times 50)$, una estructura en bandas. La resolución de las bandas oscuras a 3.000 aumentos (Fig. 4) permite observar zonas ricas en carburos, los cuales aparecen tanto en el interior como en el límite de grano. Las zonas claras, por el contrario, muestran menor 
TABLA I.- Composición química de los aceros utilizados, $\%$ en peso

TABLE I.- Chemical composition for both used steels, \% wt

\begin{tabular}{|c|c|l|}
\hline Elementos & ASTM A 533 B & F-156 \\
\hline $\mathrm{C}$ & 0,180 & 0,170 \\
$\mathrm{Mn}$ & 1,420 & 0,54 \\
$\mathrm{Si}$ & 0,240 & 0,26 \\
$\mathrm{P}$ & 0,017 & 0,005 \\
$\mathrm{~S}$ & 0,004 & 0,005 \\
$\mathrm{Cr}$ & 0,120 & 0,97 \\
$\mathrm{Ni}$ & 0,840 & 3,34 \\
$\mathrm{Mo}$ & 0,510 & 0,26 \\
$\mathrm{~V}$ & 0,002 & - \\
$\mathrm{Cu}$ & 0,140 & 0,06 \\
\hline \hline O $\sigma_{\mathrm{y}}, \mathrm{MPa}$ & 484 & 505 \\
\hline Res. Tracción, & 622 & 641 \\
$\mathrm{MPa}$ & & \\
\hline Alargamiento, $\%$ & 26 & 26 \\
\hline Estricción, \% & 77 & 70 \\
\hline
\end{tabular}

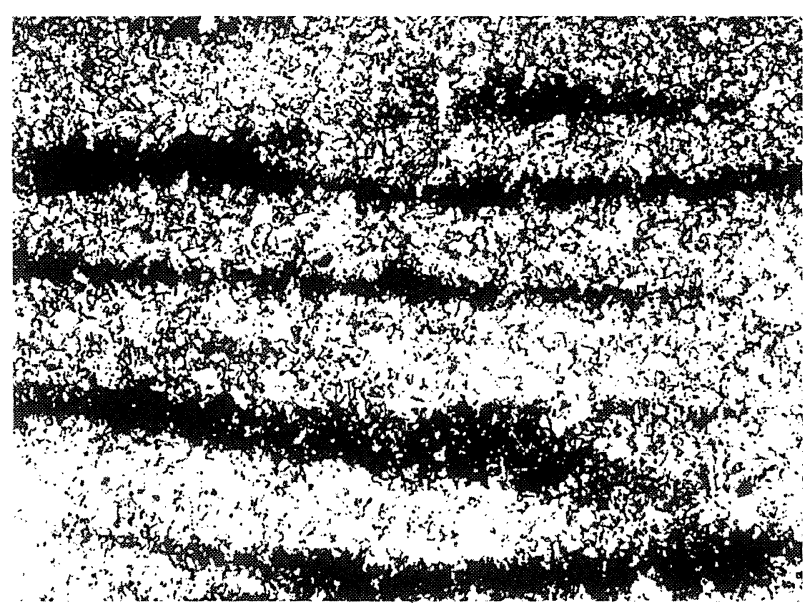

FIG. 3.- Microestructura del acero A 533 B1 $(\times 50)$.

\section{FIG. 3.- Microstructure of A 533 Bl steel (50×).}

enriquecimiento de estos precipitados. Debido a la presencia de estas zonas ricas en carburos cabe esperar una elevada dispersión de resultados mecánicos.

Para el segundo acero, al que a partir de ahora se denominará $\mathrm{B}$, aparece una estructura martensítica débilmente revenida (Fig. 5), típica de aceros de herramientas. Debido a esta microestructura cabe esperar que la tenacidad de fractura asociada de este acero sea algo mayor que para el acero de vasija A-533 B1. Esta afirmación puede también dedu-

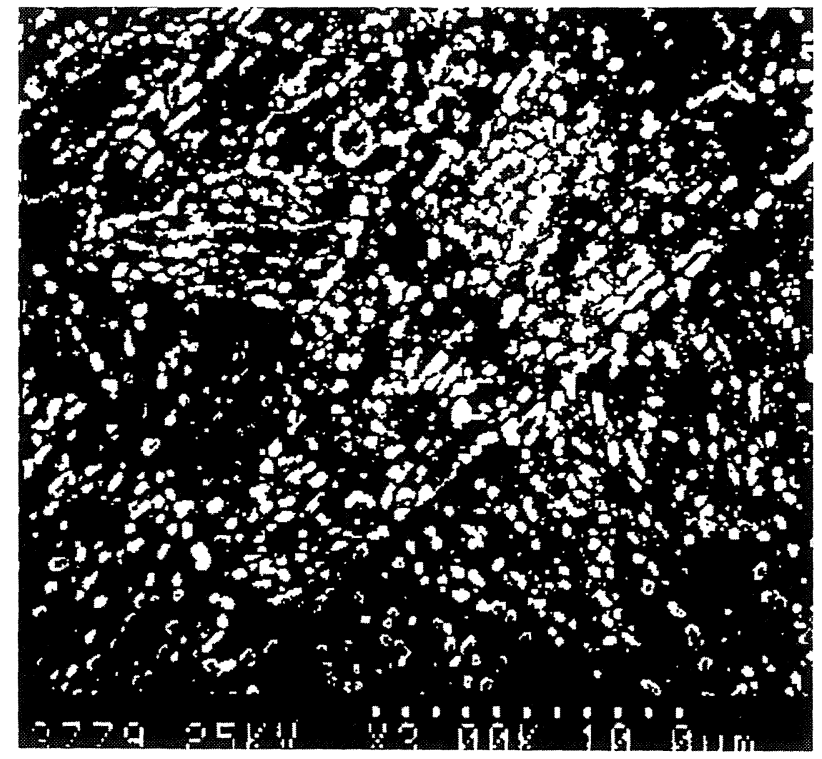

Fig. 4.-- Detalle de las bandas oscuras $(\times 3.000)$. FIG. 4.-Detail of dark bands (3.000×).

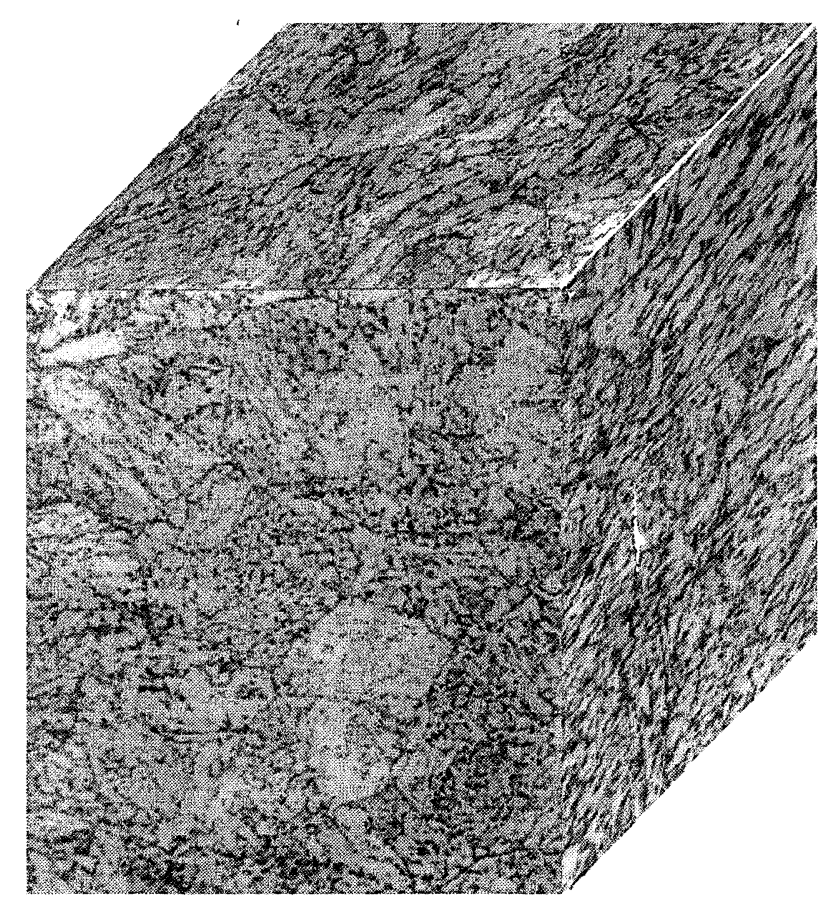

Fig. 5.- Microestructura del acero F $156(\times 500)$.

FIG. 5.- Microstructure of F 156 steel $(500 \times)$.

cirse de los datos de resistencia mecánica y límite elástico, algo superiores para el acero B.

Para la realización de ensayos cuasi-estáticos de tenacidad de fractura se ha empleado una máquina universal de ensayos MTS 810, dotada con una cámara ambiental para alcanzar un rango de temperaturas de -70 hasta $120^{\circ} \mathrm{C}$. La normativa de ensayos seguida según el comportamiento de la probeta ha sido ASTM E 399 para comportamientos marcadamente frágiles y E 813-87 en donde aparecían 
comportamientos elasto-plásticos o decididamente plásticos.

En el caso de ensayos de impacto, se ha empleado un péndulo instrumentado marca Wolpert de 300 $\mathrm{J}$ provisto de un sistema de adquisición de datos de $1 \mathrm{MHz}$ de resolución, ya que para la interpretación de ensayos en péndulo instrumentados no existe una normativa aceptada se han seguido las directrices comunes para ensayos Charpy de la norma ASTM E 23. Las probetas utilizadas han sido Charpy estándar (entalla en V).

Previamente a la realización de los ensayos, ambas máquinas han sido debidamente calibradas y homologadas por el departamento de metrología del centro.

\section{RESULTADOS Y DISCUSIÓN}

La metodología de análisis de datos empleada puede describirse como sigue:

- Realización de ensayo Charpy instrumentado y análisis de resultados mediante la determinación de los puntos críticos (1) como aparición de singularidad en el borde de la entalla; evolución hacia fisura de borde agudo; crecimiento estable de esta fisura; crecimiento acelerado con rotura final de la probeta (2) y cálculo de la tenacidad de fractura en la zona de interés ya determinada.

- Realización de ensayo de tenacidad de fractura con probetas CT (1 y 1/2") del mismo material a la misma temperatura que el ensayo Charpy instrumentado.

- Comparación de resultados entre ensayos dinámicos y estáticos

\subsection{Ensayos Charpy instrumentado}

Se han obtenido las curvas Charpy energía-temperatura para los dos materiales. El aspecto general de estas curvas es el que muestra la figura 6.

La señal fuerza-tiempo obtenida en Charpy instrumentado es la base para la posterior evaluación de parámetros mecánicos del material. En un registro típico, que a título de ejemplo recoge la figura 7 , se puede apreciar cómo la señal es una composición de al menos dos señales diferenciadas: de un lado la evolución de la fuerza media soportada por la probeta en función del tiempo, gráfica superior de la figura 7 , y de otro, una señal oscilante superpuesta, perteneciente a la vibración natural de la probeta tras el primer impacto del péndulo (gráfica inferior de la misma figura). Esta separación de señales, realizada mediante software de desarrollo propio, justifica los dos criterios de análisis presentados a continuación.

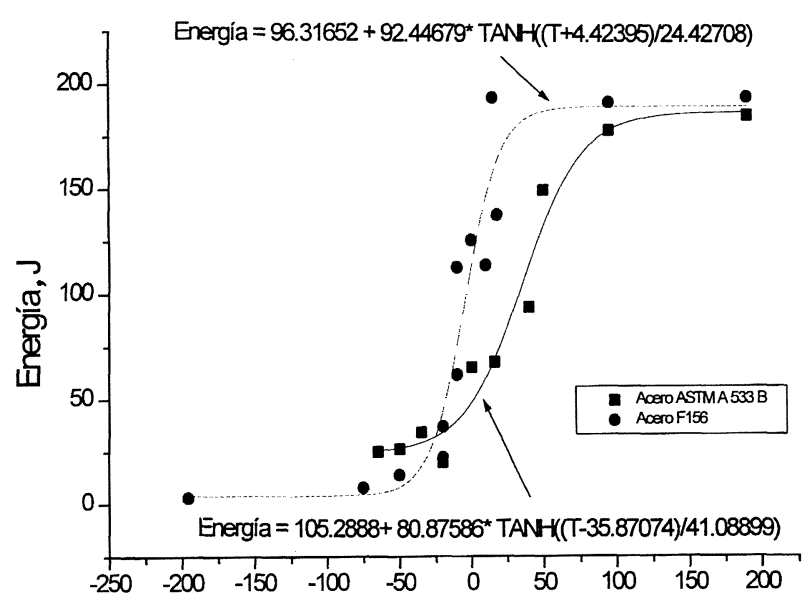

FIG. 6.- Curvas energía-temperatura para ambos aceros.

FIG. 6.- Energy \& temperature curves for both steels.

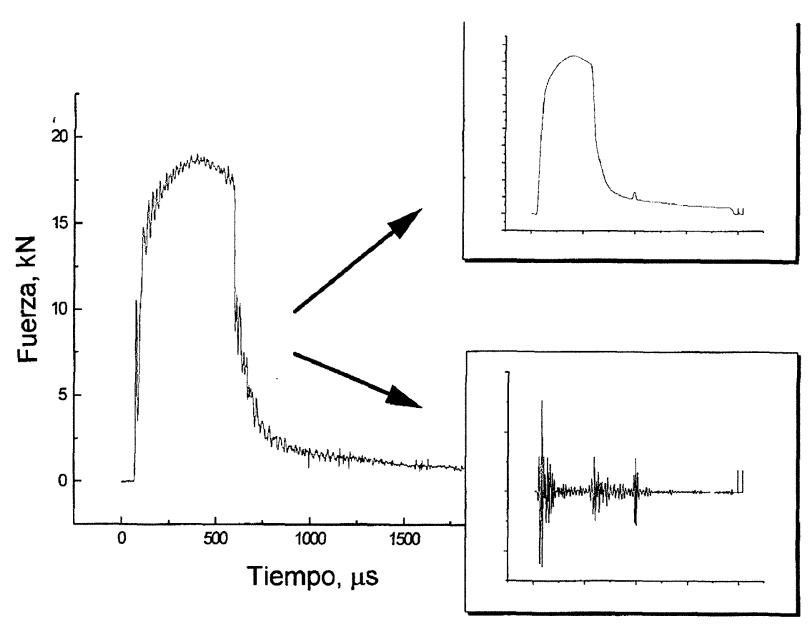

FIG. 7.- Filtrado digital de señal.

FIG. 7.-Digital signal filtering.

\subsubsection{Análisis por criterios de flexibilidad}

Utilizando probetas preagrietadas en el ensayo Charpy, el instante de crecimiento generalmente se asocia al punto de carga máxima. Por el contrario, en las probetas Charpy V estándar la iniciación de la grieta se produce generalmente antes de alcanzarse el valor de carga máxima.

Para el cálculo de la tenacidad de fractura utilizando probetas Charpy V sin preagrietar se pueden hacer dos simplificaciones sin cometer un gran error:

- Durante el régimen de deformación elástica se puede asumir la respuesta de la probeta como la de una viga de las mismas dimensiones mantenida en dos apoyos y sometida a carga central.

$$
D=\frac{P L^{3}}{48 E I_{\mathrm{z}}}
$$


- Como la tenacidad de fractura es básicamente una medida de la resistencia a la progresión de una grieta en un material, una vez nucleada la grieta, el crecimiento de la misma es lento en las etapas iniciales.

Por lo tanto, el principal problema en el análisis de los registros proporcionados por un ensayo Charpy instrumentado es determinar en qué instantes de todo el registro tiene lugar la nucleación de grieta, crecimiento estable de la grieta y crecimiento brusco final.

Estos puntos críticos de interés se han determinado empleando la relación directa que existe entre la longitud de grieta y el valor de flexibilidad de la probeta. Esta dependencia se analiza mediante la derivada de la flexibilidad en función del tiempo calculando varios puntos críticos.

Una vez filtrada la señal original eliminando la señal oscilatoria se procede al cálculo del desplazamiento mediante las siguientes expresiones:

$$
v(t)=v_{0}-\frac{\Delta t}{2 m} \sum_{\mathrm{i}=1}^{\mathrm{T}} F_{\mathrm{i}}-F_{\mathrm{i}+1}
$$

donde se obtiene la velocidad puntual del martillo y finalmente:

$$
s(t)=\frac{\Delta t}{2} \sum_{\mathrm{i}=\mathrm{t}}^{\mathrm{L}} V_{\mathrm{i}}+V_{\mathrm{i}+1}
$$

donde

$V_{0}=$ Velocidad inicial

$F=$ Fuerza

$\Delta_{\mathrm{t}}=$ Intervalo de tiempo (1/frecuencia)

$m=$ Masa de la cabeza del péndulo.

Una vez obtenido el desplazamiento $S(t)$ se obtiene la función flexibilidad. En la figura 8 se muestran curvas obtenidas sobre dos probetas de un mismo material a diferentes temperaturas sobre una función de flexibilidad.

La función de flexibilidad representada en la figura 9 para una probeta estándar presenta un máximo característico. Este máximo, trasladado a la curva original fuerza-deformación, coincide razonablemente con los criterios propuestos para la determinación de la plastificación generalizada (general yield) (3).

Una vez obtenidos los puntos críticos de las derivadas de la flexibilidad respecto al tiempo, el siguiente paso es calcular $K_{\mathrm{j}}$ a través de las siguientes expresiones:

$$
J=\frac{2 \text { energía }}{B(W-b)}
$$

donde

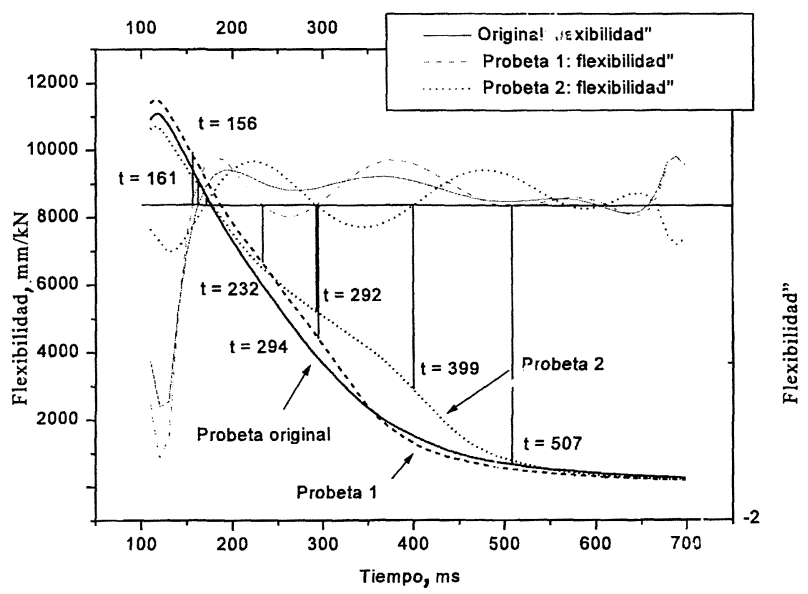

FIG. 8.- Determinación de puntos críticos para tres probetas de acero $\mathrm{A}\left(110^{\circ} \mathrm{C}\right)$.

FIG. 8-Critical points determination in three $A$ steel specimens $\left(110^{\circ} \mathrm{C}\right)$.

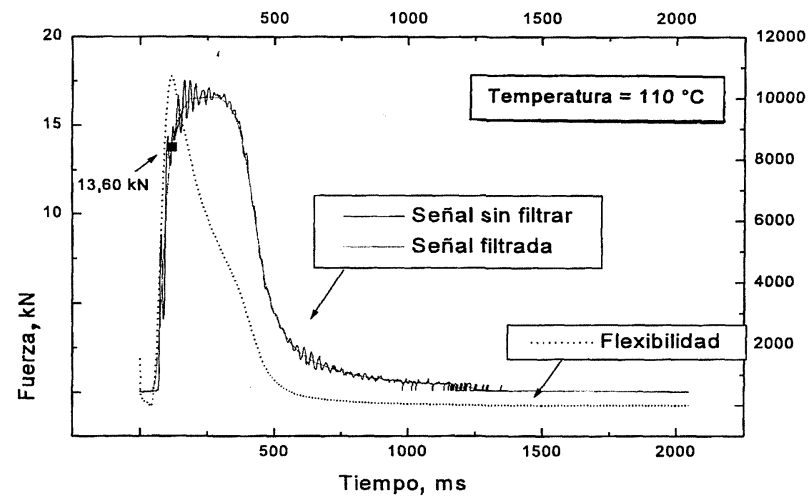

FIG. 9.- Localización de la plastificación generalizada (general yield) en el máximo de la función flexibilidad.

FIG. 9.- Location of general yield at maximum of compliance function.

$B=$ Anchura de la probeta.

$W=$ Altura de la probeta.

$b=$ Longitud de la grieta inicial.

y finalmente calculando $K_{\mathrm{j}}$ como (4):

$$
K_{\mathrm{j}}=\sqrt{J E_{\mathrm{din}}}
$$

donde

$E_{\text {din }}=$ Módulo de elasticidad.

\subsubsection{Análisis por criterios de vibración}

Tras el primer impacto del péndulo, se produce una serie de ondas de presión que recorren la probeta a una velocidad aproximada de $5.000 \mathrm{~m} / \mathrm{s}$ 
(5). Si se tiene en cuenta además que el fenómeno de vibración viene condicionado por la geometría de la probeta es bastante intuitivo pensar que cualquier cambio en la geometría (progresión de una grieta central que divide la probeta en dos partes) ha de afectar de algún modo al fenómeno oscilatiorio descrito. Atendiendo a las dos variables que definen un fenómeno oscilatorio: frecuencia y amplitud, cabe hacer un análisis separado para cada una de ellas.

\subsubsection{Frecuencia}

Se realizó un análisis de frecuencias del registro fuerza-tiempo mediante las transformadas rápidas de Fourier, obteniendo un espectro de frecuencias con su correspondiente amplitud (Fig. 10). Este procedimiento de evaluación no permitió observar claramente la aparición de la frecuencia típica de vibración de la mitad de una Charpy, ni obviamente determinar ningún punto atribuible al crecimiento estable de la fisura, por lo que se optó por abandonar definitivamente esta vía.

\subsubsection{Amplitud}

El segundo parámetro que define una onda es su amplitud. Mientras la probeta se encuentre en la región de comportamiento elástico, se puede esperar un movimiento oscilatorio con un débil amortiguamiento. Cuando empieza a deformarse, y durante el proceso de rotura, el coeficiente de amortiguamiento y la amplitud van variando. Un ejemplo de estas variaciones es el que se indica en la figura 11.

Desafortunadamente, este tipo de análisis no permite determinar cuantitativamente la progresión estable de la grieta, pues hay que tener en cuenta que el fenómeno oscilatorio descrito se propaga por

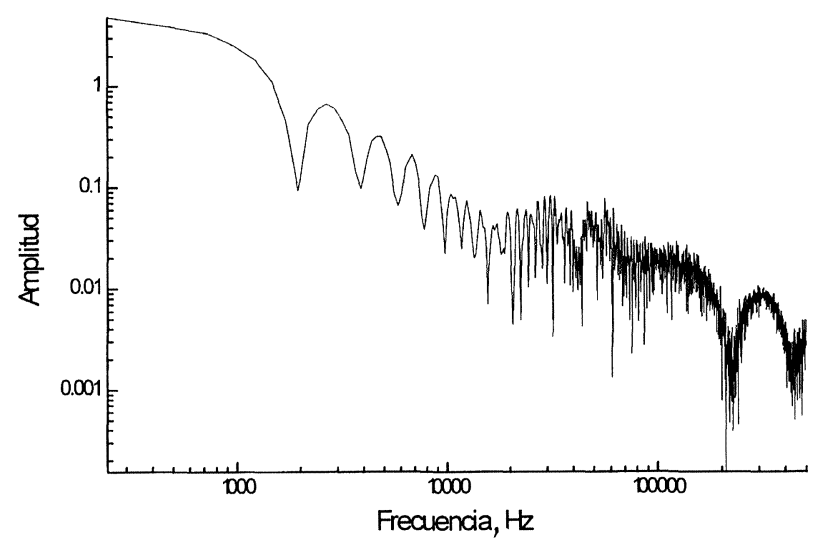

FIG. 10.- FFT de la señal original.

FIG. 10._-FFT applied to the original signal.

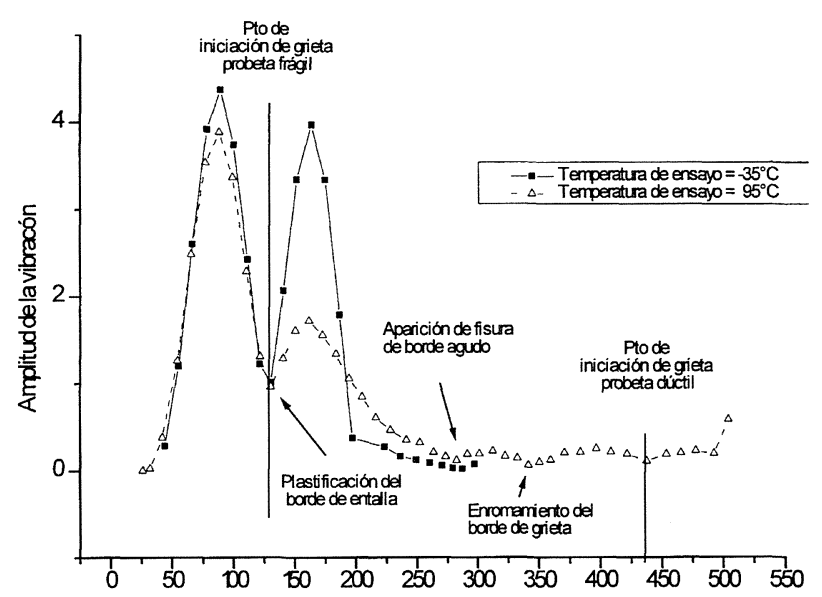

FIG. 11.- Comparación de comportamientos dúctil/frágil en un diagrama amplitud-tiempo.

\section{FIG. 11.- Comparison between ductile/brittle behaviour in an amplitude-time diagram.}

el material a una velocidad determinada, lo que provoca retrasos en la señal.

No obstante, sí es posible determinar cualitativamente en qué zona del espectro se encuentra el instante de iniciación. Así, por ejemplo, en las gráficas de la figura 12 para el acero A $533 \mathrm{~B} 1$, se aprecia que en el índice 223 se produce un mínimo en la amplitud. Según el tratamiento de la señal base por criterios de flexibilidad dicho instante ocurre en el índice 215, con lo que se detecta un retraso máximo de $8 \mu \mathrm{s}$. Este punto corresponde a una temperatura de $16{ }^{\circ} \mathrm{C}$, donde la rotura tiene un carácter mixto dúctil/frágil según la figura 6 , es decir, correspondiendo con la zona de transición. El pequeño retraso detectado señala que el borde de grieta había progresado de forma notable antes de su crecimiento final.

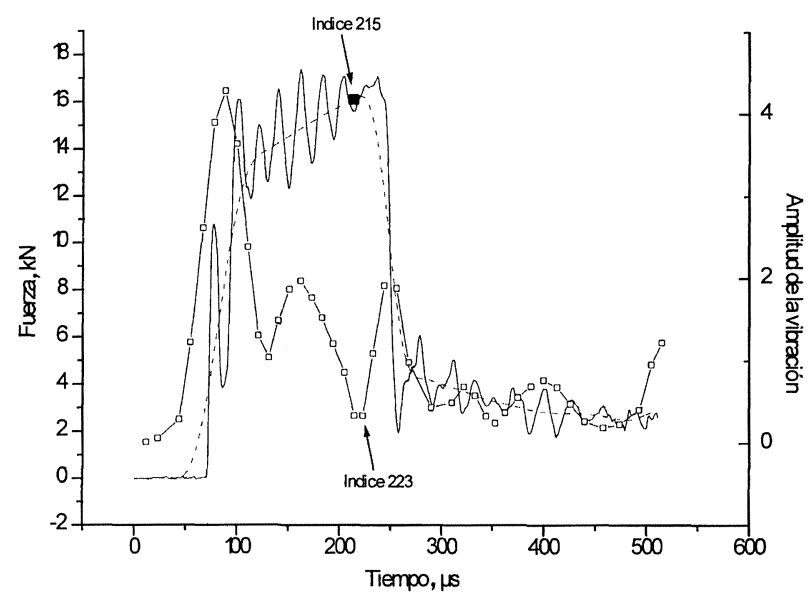

FIG. 12.- Determinación del instante de iniciación según criterios.

FIG. 12.- Evaluation of crack initiation according to criteria used. 
La gráfica de la figura 13 , obtenida a $95{ }^{\circ} \mathrm{C}$, señala un retraso de $83 \mu$ s, lo que en principio se puede esperar dado que a la temperatura señalada, los fenómenos de plasticidad han de darse en mayor extensión, permitiendo que la iniciación del crecimiento ocurra cuando la probeta ha sufrido una importante cantidad de deformación plástica.

Para estimar la longitud de la grieta sin recurrir a métodos externos como cámaras de alta velocidad o extensometría láser, se puede recurrir a un cálculo matemático basado en el efecto doppler. Hasta el momento, los cálculos efectuados en esta línea no han mostrado buenos resultados, por lo que quizás sería necesario un tratamiento previo de la señal oscilatoria pura mediante una autocorrelación.

\subsection{Ensayos estáticos}

Los ensayos de tenacidad de fractura se han llevado a cabo con arreglo a las normas ASTM E 399 y ASTM E 813.

Los datos considerados como válidos por las citadas normas son los que se han tenido en cuenta para este estudio, es decir, aquellos que cumplen la relación:

$$
B, b_{0}=25 \frac{J_{\text {ic }}}{\sigma_{\mathrm{y}}}
$$

donde

$J_{\mathrm{IC}}=$ Valor crítico de $J$ en el punto de iniciación de la grieta estable.

$\sigma_{\mathrm{y}}=$ Límite elástico efectivo del material a la temperatura de ensayo.

La representación gráfica de la figura 14 recoge estos resultados "válidos" a distintas temperaturas.

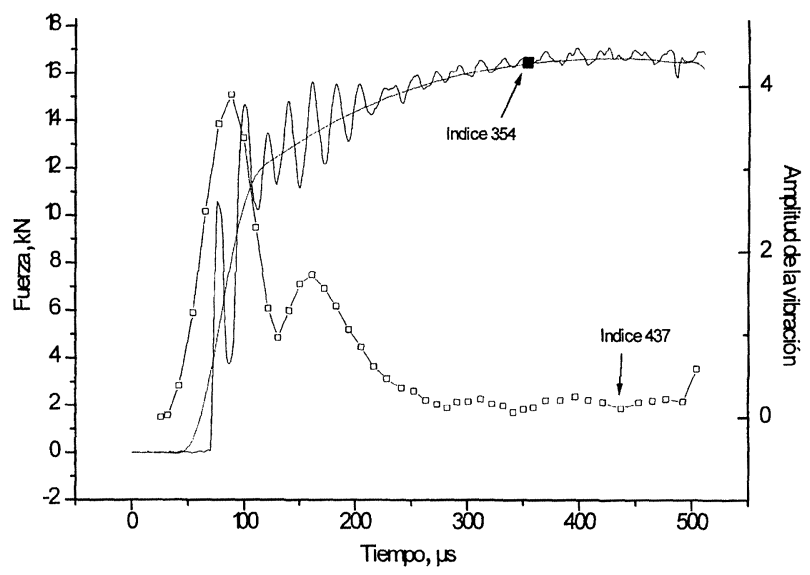

FIG. 13.- Determinación del instante de iniciación de grieta según los criterios empleados para una probeta con comportamiento completamente dúctil.

FIG. 13.- Evaluation of crack initiation according to criteria used in a completly ductile specimen.

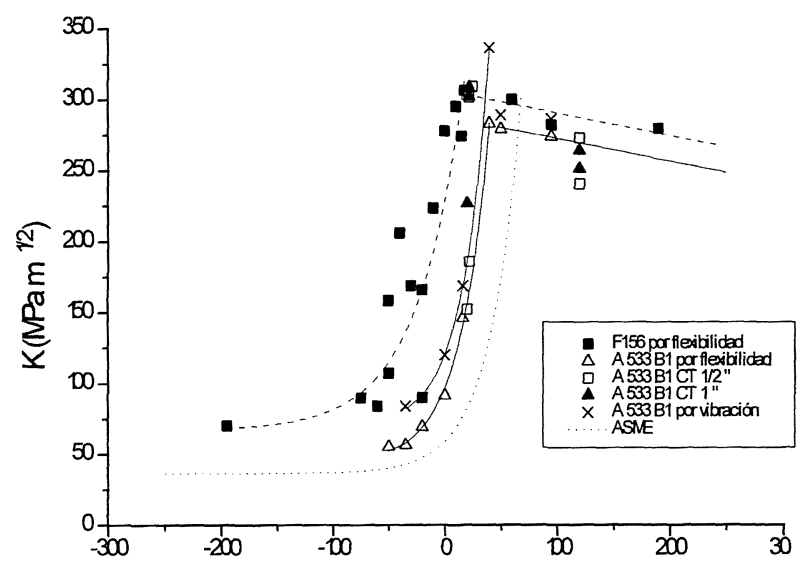

FIG. 14.- Tenacidad de fractura calculada en función de la temperatura de ensayo.

FIG. 14.- Calculate fracture toughness as a function of test temperature.

\subsection{Comparación de resultados}

En la figura 14 se representan valores de $K_{\mathrm{j}}$ obtenidos de ensayos dinámicos en función de la temperatura de ensayo para los dos materiales seleccionados y atendiendo a los dos criterios expuestos (flexibilidad y amplitud de vibración). Los resultados de $K_{\mathrm{j}}$ obtenidos se ajustan bien a expresiones tradicionalmente empleadas en la bibliografía del tipo (6):

$$
K=A+B e^{\left(C^{*} \text { temperatura }\right)}
$$

para aceros de vasija. Los valores de $K_{\mathrm{j}}$ han mostrado una pequeña dispersión aunque el ajuste para el acero A 533 B1 es notoriamente mejor que para el caso del acero F-156.

Por comparación, en la figura 14 se ha incluido también la curva ASME sección XI considerada como una envolvente inferior de los valores de $K_{\mathrm{IC}}$ para el caso de aceros de vasija. El hecho de que los valores de tenacidad obtenidos se sitúen por encima de esta envolvente parece estar de acuerdo con la proximidad encontrada a los valores de tenacidad estática. No obstante, esta condición, necesaria, aunque no suficiente, no permite asegurar que los resultados de tenacidad así obtenidos (métodos dinámicos) sean equivalentes a sus homólogos estáticos.

Igualmente, cabe señalar que la discrepancia de valores de tenacidad resultantes del empleo de ambos métodos es pequeña. No obstante, por las limitaciones existentes y comentadas en el caso de los criterios de vibración, se producen pequeñas sobreestimaciones en el valor de $K_{\mathrm{j}}$.

Como es sabido, la temperatura de ensayo afecta al módulo de elasticidad, disminuyendo su valor a medida que aumenta la temperatura. Un ejemplo de esta afirmación se recoge en la figura 15. 


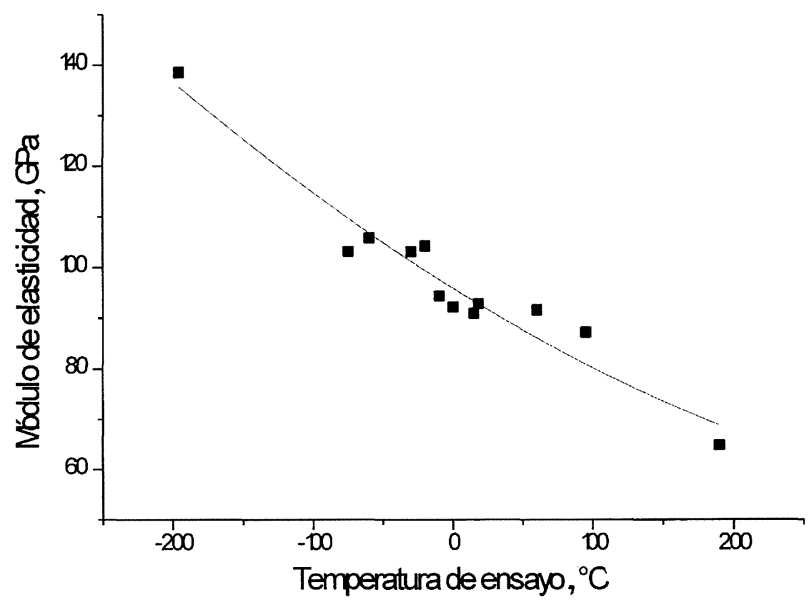

FIG. 15.- Módulo de elasticidad dinámico en función de la temperatura de ensayo.

FIG. 15.-Dynamic elastic modulus as a function of test temperature.

\section{CONCLUSIONES}

- Se ha desarrollado y verificado una sistemática de análisis para ensayos Charpy instrumentado que proporciona una herramienta de gran utilidad en la estimación de valores resistentes del material.

- Se ha podido verificar una significativa coherencia entre datos dinámicos y estáticos, clásicamente puesta en duda, y que puede abrir nuevas expectativas en su campo de aplicación (por ej. análisis más detallado de cápsulas de vigilancia, obtención de tenacidades de fractura desde ensayos Charpy con menores incertidumbres, etc.).

- Según los resultados de este análisis, parece factible obviar el preagrietamiento de las probetas Charpy V, lo que implicaría una reducción en los costes del ensayo y minimiza el tiempo de manejo de material irradiado.
- Aparte de las buenas expectativas despertadas por lo hasta ahora expuesto es completamente necesario realizar análisis estadísticos en distintos materiales y condiciones de ensayo, para delimitar los rangos de aplicabilidad de la técnica de análisis descrita.

El trabajo realizado es solamente el comienzo de un análisis más profundo, pues queda aún por determinar un buen número de variables puestas en juego en el ensayo. Una vía adicional que actualmente es objeto de estudio es el análisis modal del espectro obtenido en el ensayo. Actualmente, se está trabajando en la instrumentación de las probetas Charpy para constatar los resultados expuestos en este documento.

\section{REFERENCIAS}

(1) Fabry, A., VAn de Velde, J. IAEA Specialists' Meeting on Irradiation Embrittlement and Mitigation. Espoo (Finlandia), octubre 1995.

(2) Kobayashi, T. Yamamoto, I y Ninomi, M. Eng. Fract. Mech., 24 (5), 1986:773-782.

(3) Fabry, A. van Walle, E. Chaouadi, R. Wannijn, J.P., Verstrepen, A., Puzzolante, J.L., Van Ransbeeck, Th. y VAN DE Velde, J. RPV Steel Embrittlement: Damge modeling and micromechanics in an engineering perspective, INRNE, 1784 Sofia (Bulgaria), octubre 1993.

(4) Rauno Rintamaa. Single specimen fracture toughness determination procedure using instrumented impact test. VTT Technical Research Centre of Finland. Espoo, 1993.

(5) Instron Wolpert. Basic Principles of Measured Data Evaluation for Impact Charpy Tests on Notched and Precracked Specimens. Enero 1996.

(6) Kim Wallin. Comparison of the scientific basis of russian and european approaches for evaluating irradiation effects in reactor pressure vessels. AMES Report no. 3. Espoo. Febrero 1995. 